\title{
Replicative genetic association study between functional polymorphisms in AVPR1A and social behavior scales of autism spectrum disorder in the Korean population
}

So Young Yang ${ }^{1 \dagger}$, Soon Ae Kim ${ }^{1 \dagger}$, Gang Min Hur ${ }^{2}$, Mira Park ${ }^{3}$, Jong-Eun Park ${ }^{4}$ and Hee Jeong Yoo ${ }^{5,6^{*}}$ (D)

\begin{abstract}
Background: Arginine vasopressin has been shown to affect social and emotional behaviors, which is mediated by the arginine vasopressin receptor (AVPR1A). Genetic polymorphisms in the AVPR1A promoter region have been identified to be associated with susceptibility to social deficits in autism spectrum disorder (ASD). We hypothesize that alleles of polymorphisms in the promoter region of AVPRTA may differentially interact with certain transcriptional factors, which in turn affect quantitative traits, such as sociality, in children with autism.
\end{abstract}

Methods: We performed an association study between ASD and polymorphisms in the AVPRTA promoter region in the Korean population using a family-based association test (FBAT). We evaluated the correlation between genotypes and the quantitative traits that are related to sociality in children with autism. We also performed a promoter assay in T98G cells and evaluated the binding affinities of transcription factors to alleles of rs7294536.

Results: The polymorphisms_RS1, RS3, rs7294536, and rs10877969-were analyzed. Under the dominant model, RS1-310, the shorter allele, was preferentially transmitted. The FBAT showed that the rs 7294536 A allele was also preferentially transmitted in an additive and dominant model under the bi-allelic mode. When quantitative traits were used in the FBAT, rs7294536 and rs 10877969 were statistically significant in all genotype models and modes. Luciferase and electrophoretic mobility-shift assays suggest that the rs $7294536 \mathrm{~A} / \mathrm{G}$ allele results in a Nf-kB binding site that exhibits differential binding affinities depending on the allele.

Conclusion: These results demonstrate that polymorphisms in the AVPR1A promoter region might be involved in pathophysiology of ASD and in functional regulation of the expression of AVPR1A.

Keywords: Autism spectrum disorder, Arginine vasopressin receptor 1A (AVPR1A), Microsatellite, Single nucleotide polymorphism, Association, Promoter

\footnotetext{
* Correspondence: hjyoo@snu.ac.kr

${ }^{\dagger}$ Equal contributors

${ }^{5}$ Department of Psychiatry, Seoul National University Bundang Hospital,

173-82, Gumi-ro, Bundang-gu, Seongnam, Gyeonggi-do 463-707, South

Korea

${ }^{6}$ Department of Psychiatry, College of Medicine, Seoul National University,

Seoul, Republic of Korea

Full list of author information is available at the end of the article
} 


\section{Background}

Autism spectrum disorder (ASD) is complex neuropsychiatric developmental disorders characterized by social communication impairments and restricted interests [1]. In spite of heterogeneous etiology, molecular genetic studies for ASD have identified susceptible candidate genes, such as those involved in neurobiological pathways, chromatin remodeling, protein translation, actin dynamics, and synaptic functions $[2,3]$.

Arginine vasopressin receptor $1 \mathrm{~A}$ (AVPR1A), a receptor for arginine vasopressin (AVP), is coupled to the $G_{\alpha q / 11}$ G-protein coupled receptor and is activated by binding AVP via phospholipase $C$. The subsequent activation of protein kinase $\mathrm{C}$ helps mediate neuronal development, memory formation, synaptic plasticity, and neuronal survival $[4,5]$. Animal and human physiobiological studies have also demonstrated that social behavior is affected by AVPR1A [6-8]. Furthermore, AVP and AVPR1A have been shown to play crucial roles in the pathophysiology of psychiatric disorders such as anxiety, depression, and post-traumatic stress disorder in rodents $[9,10]$. AVPR1A signaling has also been shown to be involved in human social and emotional behavior as demonstrated in a study using the AVPR1A antagonist SRX246 [9]. Furthermore, administration of AVP via a nasal spray has been shown to improve social recognition of emotionally balanced faces in humans [11]. The variations in the gene structure of AVPR1A mostly occur in the form of genetic polymorphisms, which have been proposed to underlie inter- and intra-specific differences in expression levels $[12,13]$ and spatial expression patterns in the brain. The promoter region of AVPR1A includes a number of highly polymorphic microsatellites, such as RS1 and RS3 [14]. Furthermore, genetic variations in the promoter region of AVPR1A have also been associated with risks for autism, in which social deficits are the major symptoms, as well as autism phenotypes in nonclinical populations [15-19]. Previous association studies from various populations have been focused on examining association of RS 1, RS3, and a few SNPs resulting in inconsistent results [20]. For instance, Tansey et al. [13] demonstrated that the shorter alleles of RS1 had weaker associations with autism in the Irish population. One recent study reported association of RS1 and RS3 with repetitive behaviors, but not diagnosis of ASD [21]. Both RS1 and RS3 showed differences in relative promoter activity as measured in the human neuroblastoma cell line, SH-SY5Y, with the shorter repeat alleles of RS1 and RS3 exhibiting decreased relative promoter activity [13]. Meyer-Lindenberg et al. [22] also reported abnormal amygdala signaling is involved in the pathophysiology of autism. Our previous studies have also shown statistically significant associations between ASD and the shorter alleles of RS1 and RS3, which have been reported to be over-transmitted as risk alleles in other populations [23]. We have also previously reported a statistically significant association of ASD with the single nucleotide polymorphisms (SNPs) rs10877969 and rs7294536 in the promoter region of AVPR1A [24].

Overall, the first objective of this study was to validate our previous association study between ASD and the polymorphisms RS1, RS3, rs7294536, and rs10877969 in AVPR1A with samples from independent cohorts from our previous studies. We analyzed quantitative correlation between genotypes and quantitative traits in probands with ASD. For these analyses, we selected quantitative traits which reflect sociality directly among various inventories used for phenotypic measures of the subjects. The second is to determine the effects of the polymorphisms on the expression level of AVPR1A. Therefore, we used luciferase assays to ascertain the functional sequence polymorphisms in the promoter region in T98G cells. We also use electrophoretic mobility-shift assay (EMSA) to evaluate how the promoter polymorphisms affect nuclear protein-binding affinities. For this, we analyzed Korean family trios composed of subjects with ASD and their biological parents, diagnosed by pervasive developmental disorder based on DSM-IV-TR diagnostic criteria.

\section{Methods \\ Subjects}

Probands and their biological parents were ascertained for ASD based on the DSM-IV-TR diagnostic criteria. To confirm the ASD diagnosis, the parents or caregivers were interviewed with the Korean version of Autism Diagnostic Interview-Revised (K-ADI-R) [25], and the probands were assessed with the Korean version of the Autism Diagnostic Observation Schedule (K-ADOS) [26]. We assessed "social ability and other behavioral symptoms of the probands by using the Social Communication Questionnaires (SCQ) [27], Asperger Syndrome Diagnostic Scale (ASDS) [28], Social Responsiveness Scale (SRS) [29], and Korean Child Behavior Checklist (K-CBCL) [30]." The Korean versions of the Social Maturity Scale (K-SMS) [31], Vineland Adaptive Behavior Sales-Interview (VABS) [32], and of Korean Educational Development Institute-Wechsler Intelligence Scale for Children-Revised (KEDI-WISC-R) [33] were used for examining adaptive functioning and intelligence. We only included subjects showing clear phenotype of ASD by clinical best estimate diagnosis by two board certified psychiatrists, based on those diagnostic instruments and measures. For diagnostic validity and reliability, the age of the probands was limited to 36 months or older. We did not put any limitation for gender. For the probands suspected to have known genetic or neurological anomalies, including Down syndrome, fragile X syndrome, tuberous sclerosis, and karyotyping and neurological examinations were performed in the screening stage to identify and exclude those subjects from further analyses. 
The subjects either parent has declared he or she has non-Korean ethnicity were excluded from enrolment. Written informed consent was obtained from the biological parents or caregivers. This study was approved by Seoul National University Bundang Hospital Institutional Review Board (IRB_B-1406/253-001).

\section{Genotyping}

Blood samples in EDTA tubes were kept at $-70{ }^{\circ} \mathrm{C}$ before use. Genomic DNA was extracted from blood using QIAamp DNA Blood kit (Qiagen, Seoul, Korea). Amplifications of the RS3 [Complex (CT) $)_{4}$-TT- $(\mathrm{CT})_{8^{-}}$ $(\mathrm{GT})_{24}$ ] and RS1 [(GATA $)_{n}$ tetranucleotide repeats] were performed according to method reported by Yang et al. (2010) (Additional file 1: Table S1 shows this in more detail). The size of the labeled PCR products was determined by capillary electrophoresis on an ABI 3100 sequencer using Gene Scan Software 2.02 (ABI, Foster city, CA, USA). Five alleles of RS3 were found in all participants in multiples of $2 \mathrm{bp}$, and there were six alleles of RS1 with multiples of $4 \mathrm{bp}$.

Two SNPs, rs7294536 and rs10877969, were genotyped using sets of primers and probes (BMS, Seoul, Korea) (Additional file 1: Table S1 shows this in more detail). PCRs were performed with Real-time Master Mix (Toyobo, Osaka, Japan) following proper reaction conditions on the realtime PCR machine (BMS, Seoul, Korea). The genotyped data was collected and the Mendelian inheritance errors for each individual polymorphism were checked by PedCheck (v.1.1) to verify the data quality and to identify any genotyping error. Detail procedure was descripted in Additional file 1: Supplementary Materials and Methods.

\section{Luciferase assay}

We used Tfsitescan (http://www.ifti.org/) to predict rs7294536 variants present within the $\mathrm{Nf}-\mathrm{kB}$ consensus sequence (5'-GGGRNNYYCC-3'). A 1641 bp fragment encompassing -108 to -1749 bp from of the $5^{\prime}$ regulatory region of $A V P R 1 A$ was amplified via PCR using human DNA (Additional file 1: Table S1 shows this in more detail). The primers used for cloning AVPR1A contained KpnI and $B g l \mathrm{II}$ linkers. The PCR products were first digested by KpnI and $B g l \mathrm{II}$ and then were cloned into the multiple cloning site of the pGL3 reporter vector (Promega, Madison, WI, USA). The rs7294536 A/G (-1502 bp) and rs10877969 A/G (-649 bp) variants were generated using the QuickChange Site-Directed Mutagenesis Kit (Stratagene, LA Jolla, CA, USA) with each primer set (Additional file 1: Table S1 shows this in more detail). For all four haplotype constructs, the pA-A construct was subsequently used as a template to generate additional possible haplotype constructs (i.e., pA-A, pA-G, pG-A, and pG-G). The pGL3-SV40-AVPR1A construct (encompassing -108 to -1749 bp from the $5^{\prime}$ regulatory region of
AVPR1A), pRLCMV-renilla, pCMV-p65, and pGL3-basic vectors were used to transfect T98G cells, which is a human glioblastoma multiforme cell line, using FuGENE HD (Roche, Mannheim, Germany). Cell lysates were assessed using the Dual-Glo Luciferase Assay System (Promega, Madison, WI, USA) in a Lumat LB 9507 luminometer (EG \&G Berthhold, Bad Wildbad, Germany). Further details are described in the Additional file 1: Supplementary Materials and Methods.

\section{EMSA and western blotting}

EMSAs were used examine for any quantitative differences in the binding affinity of $\mathrm{Nf}-\kappa \mathrm{B}$ sub-family proteins to the $\mathrm{A}$ or $G$ allele of rs7294536. A nuclear extract was prepared from transfected T98G cells that were either unstimulated or stimulated with $30 \mathrm{ng} / \mathrm{mL}$ TNF- $\alpha$ for 15,30 , and $180 \mathrm{~min}$. Unlabeled double-stranded probes were annealed with equimolar concentrations of sense and antisense single-stranded probes. Radiolabeled probes were labeled with $\left[\alpha-{ }^{32} \mathrm{P}\right] \mathrm{dCTP}$ at the 2-base overhang (GG) on the 5 '-end via end labeling with a Klenow fragment and then purified through a Sephadex G50 column. During the preincubation, the binding reaction was performed with the prepared nuclear extract and unlabeled probes in the reaction buffer at room temperature. Next, p50/p65 antibodies were added for the EMSA. Thereafter, radiolabeled probe ( $1 \mathrm{ng}, 1 \times 10^{5} \mathrm{cpm}$ ) was added to the reaction mixture and then incubated for $10 \mathrm{~min}$ at room temperature. The reaction mixtures were fractionated on a non-denaturing $6 \%$ polyacrylamide gel at $200 \mathrm{v}$ for $2 \mathrm{~h}$. The gel was transferred to $3 \mathrm{MM}$ paper, dried, and then was visualized by exposing it to X-ray film. For the EMSA, $10 \mu \mathrm{g}$ of the nuclear proteins were denatured and separated on a 4-20\% SDSpolyacrylamide gel (Invitrogen, Medison, WI, USA). The fractionated proteins were then transferred to a nylon membrane (Millipore, Bedford, MA, USA), and a western blot was performed with antibodies against rabbit p50 and p65 (Santa Cruz Biotechnology, Santa Cruz CA, USA). Further details are described in the Additional file 1: Supplementary Materials and Methods.

\section{Statistical analysis}

To assess each individual polymorphism and haplotype, the transmission disequilibrium test (TDT) using the family-based association test (FBAT) program package (http://www.biostat.harvard.edu/fbat/fbat.htm) under available modes and models was used. The haplotype familybased association test (HBAT) from the FBAT program package was used to analyze associations between ASD and the haplotypes and ASD with a minimum frequency of 5\%. A permutation test of 100,000 cycles was performed using the Monte Carlo option to determine the statistical power of HBAT. In addition, the $D^{\prime}$ value between the microsatellites and SNPs was estimated with the FBAT program 
package. Statistical significance was considered at $p<0.05$ for the TDT haplotype analysis. In addition to the basic TDT and HBAT, we measured multiple quantitative traits using the FBAT program. Quantitative traits include the total score of the ADI-R diagnostic algorithm, SCQ (lifetime), and subdomain scores of ASDS, SRS, and $\mathrm{K}-\mathrm{CBCL}$ (social problems). All of the $p$ values from the FBAT in our study were subject to Bonferroni correction to account for multiple testing.

The statistical analyses for luciferase assay were conducted using a Kruskal-Wallis test, followed by Dunn's post hoc test or Mann-Whitney $U$ test. Statistical significance was considered at $p<0.05$. The relative luciferase units (RLUs) of the constructs were compared via analysis of variance (ANOVA) or unpaired Student's $t$ tests (SPSS. ver.15.0, Chicago, IL, USA).

\section{Results}

\section{Subjects}

A total of 212 families composed of affected children $(n=212)$, as well as their biological mothers $(n=212)$ and fathers $(n=209)$ were analyzed. The mean age of proband was $97.72 \pm 56.42$ months old (average \pm standard deviation). Of the probands, $84.44 \%$ were males, $87.3 \%$ had autistic disorder, $11.3 \%$ had pervasive developmental disorder not otherwise specified (PDD-NOS), and $1.4 \%$ had Asperger's syndrome as defined by the DSM-IV-TR diagnostic criteria. Clinical data of the probands for sociality traits are summarized in Additional file 1: Table S2.

\section{Genetic association study of the RS1 and RS3 microsatellite polymorphisms}

No associations were observed between RS3 and ASD in the present replication sample set, though a significant association was observed in our previous analyses [23]. The shot allele, RS1-310, was preferentially transmitted in the dominant model under the allelic mode $(Z=2.302$; $p=0.021$ ) (Table 1).

\section{Genetic association study of single nucleotide polymorphisms}

The FBAT showed that the rs10877969 A allele was significantly transmitted in all models and modes $(Z=4.796 ; p<0.001)$. The rs7294536 A allele also showed significant transmission in the additive $(Z=2.062 ; p=0.039)$ and dominant $(Z=2.043$; $p=0.041)$ models in the bi-allelic mode. These findings were consistent with our previous report regarding the transmission of AVPR1A SNPs in ASD (Table 1).

\section{Haplotype analyses}

Haplotypes were selected from different combinations of the four polymorphisms (RS1, RS3, rs7294536, and rs10877969) using a sliding window approach (frequency $<0.05)$. A significant association was found in two haplotypes of rs7294536/rs10877969, A/A $(p<0.001)$ and A/G $(p<0.001)$, under the bi-allelic mode. The RS1/ rs10877969 (310-A, $p=0.011$ ) and rs7294536/RS1/ rs10877969 (A-310-A, $p=0.012$ ) haplotype combinations were significantly associated with ASD. However, there were no statistically significant associations between any RS1/RS3 haplotype combinations in this study (Table 2).

\section{Quantitative association of the markers}

The FBAT analysis showed significant association between the SNPs (rs7294536 and rs10877969) and multiple quantitative traits related to sociality and social communication, including total scores of SCQ and SRS, social trait score of ASDS, and subdomain scores of the "qualitative abnormality in reciprocal social interaction" domain of ADI-R ( $p=0.03$ to $<0.001)$. For rs10877969, the FBAT analysis for quantitative traits was statistical significant in all models and modes. Strongly significant results $(p<0.001)$ were observed for analyses of ASDS social, SRS total, social problems in K-CBCL social problems, ADIR-A1: failure to use nonverbal behaviors to regulate social interaction, and VABS-social ability (Table 3). Furthermore, rs7294536 was weakly associated with quantitative traits $(p=0.02-0.045)$. Weak associations were observed between the social phenotypes and either microsatellite polymorphisms. In the recessive model under the bi-allelic mode, the RS3-328 allele only showed weak significance with quantitative social phenotype scores ( $p=0.02-0.036)$ (Additional file 1: Table S3 shows this in more detail).

Haplotype analyses of the quantitative traits for the RS1/SNP and SNP/SNP combinations were statistical significant. A significant effect was observed between the A/A and A/G haplotypes of rs7294536/rs10877969 for quantitative traits related to sociality in all models and modes $(p<0.05)$. The haplotype rs7294536/RS1/ rs10877969 (A/310/A) and social traits were significantly associated in the additive model under multi-allelic mode $(p<0.001)$. Results for the $\mathrm{A} / \mathrm{G}$ haplotype of rs7294536/rs10877969 was also significantly associated with social traits $(p=0.001-0.025)$.

A multi-trait test was performed for multiple traits (SCQ, ASDS, SRS, K-CBCL social problems, and VABS in bi-allelic mode and multi-allelic mode) and the rs7294536 and rs10877969 SNPs. The results from all models under the bi-allelic mode were statistically significant (additive model, $p=0.009$; dominant model, $p=0.015$; and recessive model, $p=0.015$ ) (Additional file 1: Table S4 shows this in more detail). In the recessive model analysis for the A allele, variations of ADI-A4 measured between the AG/GG allele and AA allele had statistical significant 
Table 1 Family-based association test of polymorphisms in AVPR1A promoter region

\begin{tabular}{|c|c|c|c|c|c|c|c|c|c|c|c|}
\hline \multirow[t]{2}{*}{ Marker } & \multirow{2}{*}{$\begin{array}{l}\text { Bi-allelic mode } \\
\text { Allele }\end{array}$} & \multirow{2}{*}{$\begin{array}{l}\text { Model } \\
\text { Freq. }\end{array}$} & \multirow{2}{*}{$\begin{array}{l}\text { Additive } \\
\text { N }\end{array}$} & \multirow[b]{2}{*}{ Z } & \multirow[b]{2}{*}{ p } & \multicolumn{3}{|l|}{ Dominant } & \multicolumn{3}{|l|}{ Recessive } \\
\hline & & & & & & $N$ & $Z$ & $p$ & $N$ & $Z$ & $p$ \\
\hline \multirow[t]{5}{*}{ RS3 } & 326 & 0.062 & 38 & -0.617 & 0.537 & 37 & -0.714 & 0.475 & - & - & - \\
\hline & 328 & 0.207 & 96 & 0.183 & 0.855 & 94 & -0.464 & 0.643 & 21 & 0.612 & 0.107 \\
\hline & 330 & 0.255 & 104 & -0.254 & 0.799 & 95 & -0.981 & 0.327 & 37 & 1.172 & 0.241 \\
\hline & 332 & 0.202 & 97 & 1.044 & 0.296 & 91 & 1.272 & 0.203 & 18 & -0.25 & 0.803 \\
\hline & 334 & 0.186 & 98 & 0.447 & 0.655 & 94 & -0.621 & 0.535 & 25 & 0.221 & 0.825 \\
\hline \multirow[t]{2}{*}{ rs7294536 } & A & 0.807 & 112 & 2.062 & 0.039 & 36 & 2.043 & 0.041 & 36 & -2.043 & 0.041 \\
\hline & G & 0.193 & 112 & -2.062 & 0.039 & 107 & -1.386 & 0.166 & - & - & - \\
\hline \multirow[t]{6}{*}{ RS1 } & 306 & 0.087 & 59 & 0.367 & 0.714 & 58 & 0.131 & 0.896 & 107 & 1.386 & 0.166 \\
\hline & 310 & 0.414 & 152 & 1.490 & 0.136 & 122 & 2.302 & 0.021 & 86 & -0.288 & 0.773 \\
\hline & 314 & 0.221 & 124 & -1.121 & 0.262 & 119 & -0.323 & 0.747 & 31 & -2.090 & 0.037 \\
\hline & 318 & 0.083 & 62 & -1.543 & 0.123 & 61 & -1.440 & 0.150 & - & - & - \\
\hline & 322 & 0.057 & 39 & 0.000 & 1.000 & 39 & 0.155 & 0.877 & - & - & - \\
\hline & 326 & 0.130 & 77 & 0.211 & 0.833 & 76 & 0.349 & 0.727 & 13 & -0.316 & 0.752 \\
\hline \multirow[t]{3}{*}{ rs10877969 } & A & 0.911 & 68 & 4.796 & 0.001 & 18 & 2.177 & 0.030 & 67 & 4.573 & $<0.001$ \\
\hline & G & 0.089 & 68 & -4.796 & 0.001 & 67 & -4.573 & $<0.001$ & 18 & -2.177 & 0.030 \\
\hline & Multi-allelic mode & & Df & $x^{2}$ & $p$ & Df & $x^{2}$ & $p$ & Df & $x^{2}$ & $p$ \\
\hline RS3 & & & 5 & 1.598 & 0.902 & 5 & 4.436 & 0.489 & 4 & 4.133 & 0.388 \\
\hline rs7294536 & & & 1 & 4.525 & 0.039 & 2 & 5.377 & 0.068 & 2 & 5.377 & 0.068 \\
\hline RS1 & & & 6 & 4.517 & 0.607 & 6 & 8.425 & 0.209 & 3 & 4.634 & 0.201 \\
\hline rs10877969 & & & 1 & 23.000 & $<0.001$ & 2 & 23.206 & $<0.001$ & 2 & 23.206 & $<0.001$ \\
\hline
\end{tabular}

Allele over-transmitted allele, Freq. frequency, $N$ number of informative nuclear families, $Z Z$ score from family-based association test, $D f$ degree of freedom, $X^{2} X^{2}$ statistics, $p, p$ value

Table 2 Haplotype analysis of polymorphisms in promoter region of AVPR1A

\begin{tabular}{|c|c|c|c|c|c|c|c|c|c|c|c|}
\hline \multirow[t]{2}{*}{ Marker } & \multirow{2}{*}{$\begin{array}{l}\text { Biallelic mode } \\
\text { Haplotype }\end{array}$} & \multirow{2}{*}{$\begin{array}{l}\text { Model } \\
\text { Freq. }\end{array}$} & \multicolumn{3}{|c|}{ Additive } & \multicolumn{3}{|c|}{ Dominant } & \multicolumn{3}{|c|}{ Recessive } \\
\hline & & & $\bar{N}$ & $z$ & $p$ & $\bar{N}$ & Z & $p$ & $\bar{N}$ & $z$ & $p$ \\
\hline $1-3$ & $330-310$ & 0.135 & 70 & -0.753 & 0.452 & 69 & -0.770 & 0.441 & 11 & -0.164 & 0.869 \\
\hline \multirow[t]{2}{*}{$2-4$} & $A-A$ & 0.736 & 133 & 3.826 & $<0.001$ & 55 & 3.651 & $<0.001$ & 118 & 2.655 & 0.001 \\
\hline & $A-G$ & 0.064 & 48 & -3.586 & $<0.001$ & 48 & -3.311 & $<0.001$ & - & - & - \\
\hline $1-2$ & $330-A$ & 0.235 & 109 & -0.230 & 0.818 & 99 & -0.937 & 0.349 & 33 & 1.212 & 0.225 \\
\hline $2-3$ & A-310 & 0.384 & 145 & 1.639 & 0.101 & 116 & 2.112 & 0.035 & 72 & 0.125 & 0.900 \\
\hline $3-4$ & $310-A$ & 0.367 & 149 & 2.552 & 0.011 & 124 & 2.995 & 0.003 & 72 & 0.564 & 0.573 \\
\hline $1-2-3$ & $330-A-310$ & 0.125 & 65 & -0.891 & 0.373 & 65 & -1.045 & 0.296 & 10 & 0.174 & 0.862 \\
\hline \multirow[t]{2}{*}{$2-3-4$} & A-310-A & 0.352 & 142 & 2.505 & 0.012 & 118 & 2.650 & 0.008 & 61 & 0.878 & 0.380 \\
\hline & Multi-allelic mode & & Df & $x^{2}$ & $p$ & Df & $x^{2}$ & $p$ & Df & $x^{2}$ & $p$ \\
\hline $1-3$ & & & 6 & 6.538 & 0.366 & 6 & 5.425 & 0.491 & - & - & - \\
\hline $2-4$ & & & 3 & 24.302 & $<0.001$ & 3 & 20.364 & $<0.001$ & 2 & 9.650 & 0.008 \\
\hline $1-2$ & & & 6 & 3.859 & 0.696 & 6 & 3.779 & 0.707 & 3 & 6.899 & 0.075 \\
\hline $2-3$ & & & 5 & 5.594 & 0.348 & 5 & 7.474 & 0.188 & 2 & 2.148 & 0.341 \\
\hline $3-4$ & & & 5 & 18.501 & 0.002 & 5 & 18.934 & 0.002 & 2 & 4.027 & 0.134 \\
\hline $1-2-3$ & & & 6 & 7.455 & 0.281 & 6 & 6.676 & 0.352 & - & - & - \\
\hline $2-3-4$ & & & 5 & 19.869 & 0.001 & 5 & 17.603 & 0.003 & 2 & 2.612 & 0.271 \\
\hline
\end{tabular}

1 RS3 (complex), 2 rs7294536, 3 RS1 (GATA), 4 rs10877969, $N$ number of informative nuclear families, $D f$ degree of freedom, $X^{2} X^{2}$ statistics, $p p$ value 
Table 3 Family-based association results of polymorphisms in AVPR1A with single quantitative trait by additive model

\begin{tabular}{lllllllllllll}
\hline Traits & & & SCQ & ASDS & SRS & K-CBCL & ADIR-A1 & ADIR-A2 & ADIR-A3 & ADIR-A4 & ADIR-total & VABS \\
Marker & Freq. & $N$ & $p$ & $p$ & $p$ & $p$ & $p$ & $p$ & $p$ & $p$ & $p$ & $p$ \\
\hline rs7294536 G & 0.193 & 71 & 0.049 & 0.019 & 0.033 & 0.015 & 0.016 & 0.029 & 0.030 & 0.019 & 0.021 & 0.407 \\
rs7294536 A & 0.807 & 71 & 0.049 & 0.019 & 0.033 & 0.015 & 0.016 & 0.029 & 0.030 & 0.019 & 0.021 & 0.407 \\
rs10877969 G & 0.089 & 47 & 0.001 & $<0.001$ & $<0.001$ & $<0.001$ & 0.001 & 0.001 & 0.001 & 0.002 & 0.001 & $<0.001$ \\
rs10877969 A & 0.911 & 47 & 0.001 & $<0.001$ & $<0.001$ & $<0.001$ & 0.001 & 0.001 & 0.001 & 0.002 & 0.001 & $<0.001$ \\
\hline
\end{tabular}

Freq. allele frequency, $N$ number of informative nuclear families, $p p$ value, SCQ Social Communication Questionnaires, $A S D S$ Asperger Syndrome Diagnostic Scale, $S R S$ social responsiveness scale, $K$ - $C B C L$ Korean Child behavior checklist (social problems), $K$ - $A D I R$ Autism Diagnostic Interview-Revised, $A D I-A 1$ failure to use nonverbal behaviors to regulate social interaction, $A D I-A 2$ failure to develop peer relationship, $A D I-A 3$ lack of shared enjoyment, $A D I-A 4$ lack of socioemotional reciprocity, VABS Vineland Adaptive Behavior Scales

differences $(t=2.434 ; p=0.016)$ (Additional file 1 : Table S5 shows this in more detail).

\section{Luciferase assay}

The relative luciferase activity for the promoter with the rs7294536 A allele was significantly higher than that of the G allele. However, rs10877969 allelic variants did not influence promoter activity (Fig. 1a).

\section{Analyses of transcriptional factor binding site}

To investigate whether the putative $\mathrm{Nf}-\mathrm{\kappa B}$ involvement is due to a transcriptional event in the AVPR1A promoter region, we synthesized 22-mer oligonucleotide probes with adjacent sequences of rs7294536 and then performed an EMSA with radiolabeled DNA. From the luciferase assays, the promoter activities of each construct were measured under normal conditions without the activation of any pathways. Therefore, first, EMSA
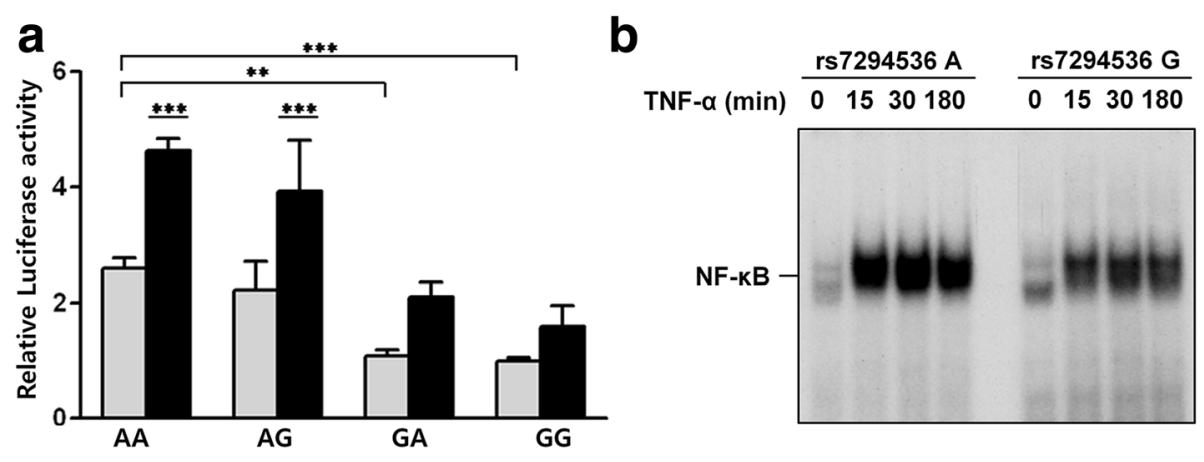

C

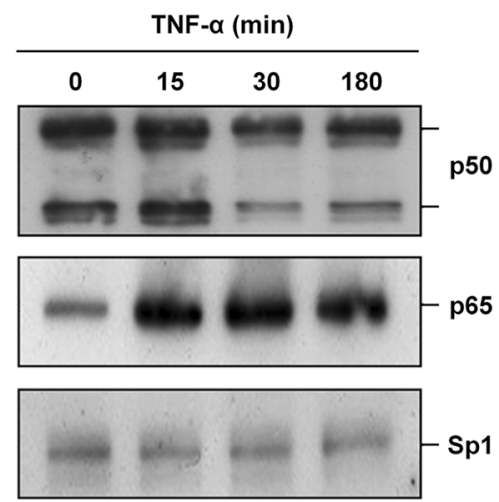

d
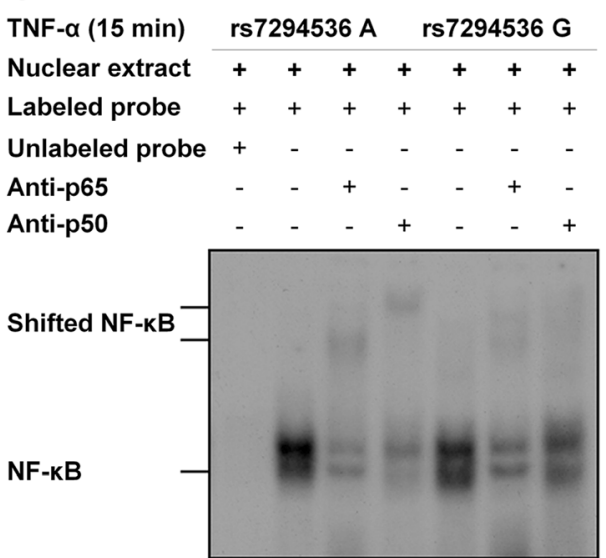

Fig. 1 Luciferase assays and electrophoresis mobility-shift assay (EMSA). a Luciferase assays with vector constructs which were contained polymorphic sites, rs7294536 (-1502 A/G) and rs10877969 (-649 A/G) in promoter region of the AVPR1A. The RLU values were denoted by mean and error bars by \pm SD $(n=6)$. ${ }^{* *} p<0.001,{ }^{* *} p<0.01$. b EMSA with oligonucleotides containing the rs7294536A or rs7294536G allele, c Western blotting with nuclear extract of T98G induced by $30 \mathrm{ng} / \mathrm{ml}$ of TNF-a. d Supershift assays with p65 and p50 antibodies and nuclear extract induced by $30 \mathrm{ng} / \mathrm{ml}$ TNF-a for $15 \mathrm{~min}$ 
was performed with the nuclear extracts of $1,3,5,10$, and $20 \mu \mathrm{g}$ in un-induced conditions for the Nf-kB pathway. We observed that the probe containing the rs7294536 G allele had a stronger binding affinity to unknown and nonspecific proteins than the A allele. To induce the $\mathrm{Nf}-\mathrm{kB}$ pathway, the cells were treated with TNF- $\alpha(30 \mathrm{ng} / \mathrm{ml})$ for $0,15,30$, or $180 \mathrm{~min}$. The nuclear extracts of the cells that were stimulated for $30 \mathrm{~min}$ showed the strongest band intensity. Thereafter, the nuclear proteins from this time point were used. Stimulating the cells with TNF- $\alpha$ resulted in a significant timedependent increase in binding between the nuclear proteins and the probe when compared with the unstimulated cells. The binding of nuclear proteins to probes for the rs7294536 A and G alleles were discriminatively detected by EMSA. A higher intensity band was observed for the probe containing the rs7294536 A allele than the rs7294536 G allele (Fig. 1b). To verify the quantity of nuclear proteins used in the EMSA, we performed a western blot analysis for p50 and p65, and SP-1 was used as an internal control. The levels of p65 and p50 in the nuclear extract of the cells stimulated with TNF- $\alpha$ for 15 and $30 \mathrm{~min}$ were increased when compared to the unstimulated cells, and SP-1 was detected uniformly (Fig. 1c). To confirm the specificity for the binding of nuclear proteins to each probe, a competition experiment was performed by mixing the nuclear proteins with anti-p50 and anti-p65 antibodies before treating them with the labeled probes. We observed supershifted bands for p65 and p50 in the competition assay (Fig. 1d). Overall, this study suggests that the rs7294536 A/G polymorphisms affect the Nf- $\mathrm{kB}$ binding site affinity. Furthermore, these results demonstrate that SNPs in the promoter region of $A V P R 1 A$ are indeed involved in regulating the expression of the AVPR1A.

\section{Discussion}

Though comparisons between AVPR1A microsatellites and social behaviors have been previously reported in animal models, the associations of specific markers with ASD have not yet been replicated. Results from a previous association study between families with ASD and RS1 and RS3 in AVPR1A have been inconsistent with our previous study [23] as well as others' studies. Kim et al. [15] first found nominally significant transmission disequilibrium of $332 \mathrm{bp}$ in RS3 in 115 non-ethnic-matched ASD families but not by a low power in Bonferroni correction. Next, Wassink et al. [16] replicated an association test between the RS1 and RS3 microsatellites in Caucasian families with autism and found a strong transmission disequilibrium for 328 bp in RS3 and RS1-(GATA) 9 in the "normal language" subgroup families. Yirmiya et al. [17], who studied 114 Israeli families, found a significant association with moderate LD between ASD and new markers located in the intron of $A V P R 1 A$.
The differences between these studies in regard to preferentially transmitted alleles may be due to the different ethnic groups that were studied and the varied statistical methodologies or sample size. In this study, our failure to replicate the genetic associations between microsatellite markers in AVPR1A and Korean individuals with ASD from our original study could be due to a several reasons. Firstly, our original findings might be false positives from lack of replication. Next, it can be possible that there is lack of adequate power to detect or replicate an association result because of lack of adequate sample size for the analysis of multiple alleles. We also cannot rule out the possibility that microsatellites frequently inherited by de novo alterations can also affect susceptibility [34]. Moreover, clinical heterogeneity of ASD must be considerable factor for replication failure.

Previously, we have suggested that microsatellites and SNPs of AVPR1A might be genetically involved in ASD [23, 24]. Moreover, this study also suggested that the social trait values among families with ASD were significantly associated with their genotypes. To the best of our knowledge, there has been no other replication study for AVPR1A polymorphisms in independent cohorts within the same population. This family-based association study replicated the results that suggested rs7294536 $\mathrm{G}$ and rs10877939 $\mathrm{G}$ alleles of $A V P R 1 A$ have been over-transmitted as risk allele in families with ASD. A significantly low $p$ value for rs10877939 and a moderate $p$ value for rs7294536 in the FBAT were observed. These results are consistent with previous reports with other ethnicities. Tansey et al. [13] showed an association between autism and the SNPs in the AVPR1A promoter, demonstrating a single association with $A V P R 1 A$ in an Irish autism trio collection with a corrected $p$ value of 0.01 for rs11174815, a tag SNP located 143 bp downstream from rs10877969. However, this group concluded that a lack of a relationship between this marker and autism in the Irish autism sample population was probably due to the very low minor allele frequency $(\mathrm{MAF}=0.015)$.

In the pathogenesis of ASD, it is assumed that multiple gene variants may be involved, each making small quantitative contributions to the final phenotype [35]. Reciprocal social interaction is one of the representative final phenotypes of ASD that is affected by genetic variants. In this study, two SNPs in AVPR1A showed significant quantitative associations with multiple measures of social behaviors and reciprocity both in current (ASDS, SRS, and VABS) and past developmental periods (ADI-R and SCQ), similar to that reported by Yirmiya et al. [17]. It also supports the notion that polymorphisms in the promoter region of AVPR1A might contribute to the dysregulation of social behavior, rather than the development of ASD itself, 
though studies in this area has been largely inconsistent between studies [36-39].

In addition, the functional role of the RS1 and RS3 microsatellites in AVPR1A has also been previously demonstrated by luciferase assays [13]. The shorter alleles of RS1 were hypothesized to increased susceptibility to autism as a result of decreased AVPR1A expression. However, compared to microsatellites, few studies have studied the function of SNPs in this region. Here, we investigated the potential role of the rs7294536 and rs10877969 SNPs in the promoter region by examining their effect on relative promoter activity and transcriptional factor interactions. We focused on rs7294536 since it is located in the recognition sequences for the $\mathrm{Nf}-\mathrm{kB}$ transcription factors, which is expected to bind with p65. The construct with the rs7294536 A allele showed higher relative promoter activity, especially when Nf-kB pathway was overexpressed. Through the EMSA, it was confirmed that the Nf- $\mathrm{B}$ binding affinities varied according to the rs7294536 alleles.

In our previous study, the rs7294536 A allele was over-transmitted in families with ASD and was considered a risk allele for ASD [24]. However, the rs7294536 A allele was observed to have higher relative promoter activity, which was counter to our hypothesis that lower promoter activity was related to autism-related deficits of behavior [12, 13]. This may be due varying AVPR $1 A$ expression levels and transfection efficiencies in the T98G cells used in this study. The T98G cell line is nonneuronal since it originated from human glioblastoma multiforme. Therefore, this can result in experimental inconsistencies with our previous study where the SHSY5Y cell line was used instead.

Secondly, our experimental setup for the promoter activity study is reminiscent of inflammatory situations $[40,41]$. The expression of IL-1 $\beta$, IL-6, IL-17, and TNF inflammatory molecules is increased in the brain, cerebrospinal fluid, and serum of some patients with ASD, whereas NF-kB is activated in the brain, which then stimulates peripheral blood immune cells in patients with autism [42-44]. Nf-kB has been recognized as a member of the Rel transcription factor family and the most widely studied form of NF- $\mathrm{kB}$ is a heterodimer of the p50 and p65 subunits, which functions as a potent activator of gene transcription [40]. Moreover, neurons, astrocytes, and microglia from patients with ASD revealed higher expression of the p65 subunit of NF- $\mathrm{kB}$ as compared with matched controls. Therefore, NF- $\mathrm{kB}$ signaling was prominent in the interacting gene networks that were constructed from the brains of patients with ASD [45]. We hypothesized that elevated NF- $\mathrm{kB}$ might result in the protection of neuronal connectivity and signaling network due to the elevated AVPR1A expression. In this study, increased differences in the relative promoter activities of the rs7294536 A and rs7294536 G alleles were observed when cells overexpressed Nf-kB or treated with TNF- $\alpha$ as compared with untreated condition. This was also observed via EMSA by the differences in binding affinities between the rs7294536 A and rs7294536 G alleles.

This study could provide novel insight into the transcriptional regulation of $A V P R 1 A$ and the potential biological roles of its gene product. Therefore, the role of AVPR1A polymorphisms should be explored with regard to the neurobiology of ASD, especially in the context of NF-kB signaling pathway. To investigate its molecular biological aspects, the expression pattern for the rs7294536 A/G alleles in AVPR1A in several cell types and specific brain regions of different organisms will be required. Furthermore, AVPR1A expression in the brain, the related polymorphic alleles, and subsequent activation of NF- $\mathrm{kB}$ signaling during inflammation will be needed to corroborate this study.

Although single predictable genetic markers were uninformative, multiple reports with accumulating evidence have demonstrated genetic associations with ASD [46]. Moreover, the carefully selected sets of critical genes should be able to be applied to sub-groups of ASD as classified by age, sex, severity, and phenotypic traits [47]. Overall, we identified a functional polymorphic site in AVPR1A that may be possibly important for socialization in ASD, and evidence was provided for its mechanistic biological role in the present study. The limitations of this study are; first, we only assessed two polymorphisms that were frequently observed in patients with ASD in this study. However, it is also possible that other undetected polymorphisms may have overt function with rs7294536 in the LD region, as there may be high LD with variants not tested. Second, the reason for inconsistency in the association analyses in microsatellite polymorphisms between current study and our previous report are not fully explained. Lastly, as the behavioral characteristics of the parents are not evaluated, there was limitation in examining relationship between AVPR1A variants and behavioral functioning in the context of broader autism phenotype.

\section{Conclusion}

In this family-based association study, we validated our previous study between $\mathrm{ASD}$ and the polymorphisms in AVPR1A with independent samples. Also, we observed quantitative correlation between genotypes and quantitative traits in probands with ASD. Luciferase and electrophoretic mobility-shift assays exhibited differential binding affinities depending on the allele of associated SNP. These results demonstrate that polymorphisms in the $A V P R 1 A$ promoter region might be involved in pathophysiology of ASD and in functional regulation of the expression of $A V P R 1 A$. 


\section{Additional file}

Additional file 1: Supplementary Materials and Methods, Table S1, Table S2, Table S3, Table S4, Table S5, Table S6, and Table S7. Details of genotyping, luciferase assay, and electrophoretic mobility-shift assay are described as text. Supplementary tables include primers and probes used in this study (Table S1), description of social traits for ASD probands (Table S2), additional results of family-based association of polymorphisms in AVPR1A with single quantitative trait by additive model (Table S3). FBAT results with multi-trait such as SCQ, ASDS, SRS, K-CBCL, and VABS (Table S4), statistical analysis of the social behavior scores in subjects with ASD with genotypes for rs 10877969 and rs7294636 (Table S5), genotype and allele frequency of rs10877969 in dbSNP b126 chr12:61,833,506.61833506 of various population (Table S6), and comparison of previous association studies for AVPR1A polymorphisms in ASD (Table S7).(DOCX 62 kb)

\section{Abbreviations}

ADI-A1: Failure to use nonverbal behaviors to regulate social interaction; ADIA2: Failure to develop peer relationship; ADI-A3: Lack of shared enjoyment; ADI-A4: Lack of socioemotional reciprocity; ANOVA: Analysis of variance; ASD: Autism spectrum disorder; ASDS: Asperger Syndrome Diagnostic Scale; AVP: Arginine vasopressin; AVPR1A: Arginine vasopressin receptor 1A; Df: Degree of freedom; EMSA: Electrophoretic mobility-shift assay; FBAT: Family-based association test; HBAT: Haplotype family-based association test; IRB: Institutional Review Board; K-ADI-R: Korean version of the Autism Diagnostic Interview-Revised; K-ADOS: Korean version of the Autism Diagnostic Observation Schedule; K-CBCL: Korean Child Behavior Checklist; KEDI-WISC-R: Korean Educational Development Institute-Wechsler Intelligence Scale for Children-Revised; K-SMS: Korean version of the Social Maturity Scale; MAF: Minor allele frequency; PDD-NOS: Pervasive developmental disorder that were not otherwise specified; RLUs: Relative luciferase units; SCQ: Social Communication Questionnaires; SNPs: Single nucleotide polymorphisms; SRS: Social Responsiveness Scale;

TDT: Transmission disequilibrium test; VABS: Vineland Adaptive Behavior Sales-Interview

\section{Acknowledgements}

Not applicable.

\section{Funding}

This work has been supported by the Healthcare Technology R\&D project (no. HI12C0021) by the Ministry of Health and Welfare, Republic of Korea; the National Research Foundation of Korea (NRF) grant funded by the Korea government (MSIP) (NRF-2014R1A2A1A110 53289 and NRF-2017M3C7A1027467).

\section{Availability of data and materials}

Not applicable.

\section{Authors' contributions}

HJY conceived the study. SYY and GMH performed the experiments. SSY, MP, and JEP analyzed the experimental data. HJY evaluated the clinical data. SYY, HJY, and SAK wrote the manuscript. All authors read and approved the final manuscript.

\section{Ethics approval and consent to participate}

Written informed consent was obtained from the biological parents or caregivers. This study was approved by Seoul National University Hospital Institutional Review Board (IRB_B-1406/253-001).

\section{Consent for publication}

The authors consent to publication.

\section{Competing interests}

The authors declare that they have no competing interests.

\section{Publisher's Note}

Springer Nature remains neutral with regard to jurisdictional claims in published maps and institutional affiliations.

\section{Author details}

'Department of Pharmacology, School of Medicine, Eulji University, Daejeon, Republic of Korea. ${ }^{2}$ Department of Pharmacology, College of Medicine, Chungnam National University, Daejeon, Republic of Korea. ${ }^{3}$ Department of Preventive Medicine, School of Medicine, Eulji University, Daejeon, Republic of Korea. ${ }^{4}$ Animal Genomics and Bioinformatics Division, National Institute of Animal Science, Wanju, Jeonbuk, Republic of Korea. ${ }^{5}$ Department of Psychiatry, Seoul National University Bundang Hospital, 173-82, Gumi-ro, Bundang-gu, Seongnam, Gyeonggi-do 463-707, South Korea. ${ }^{6}$ Department of Psychiatry, College of Medicine, Seoul National University, Seoul, Republic of Korea.

Received: 6 April 2017 Accepted: 4 August 2017

Published online: 09 August 2017

\section{References}

1. American Psychiatric Association. Diagnostic and statistical manual of mental disorders (5th ed)-DSM 5. Arlington: American Psychiatric Publishing; 2013.

2. Betancur $\mathrm{C}$. Etiological heterogeneity in autism spectrum disorders: more than 100 genetic and genomic disorders and still counting. Brain Res. 2011; 1380:42-77

3. Bourgeron T. Current knowledge on the genetics of autism and propositions for future research. C R Biol. 2016;339:300-7.

4. Thibonnier M, Auzan C, Madhun Z, Wilkins P, Berti-Mattera L, Clauser E. Molecular cloning, sequencing, and functional expression of a cDNA encoding the human V1a vasopressin receptor. J Biol Chem. 1994;269:3304-10.

5. Caldwell HK, Lee HJ, Macbeth AH, Young WS. Vasopressin: behavioral roles of an "original" neuropeptide. Prog Neurobiol. 2008;84:1-24.

6. Insel TR, Wang ZX, Ferris CF. Patterns of brain vasopressin receptor distribution associated with social organization in microtine rodents. J Neurosci. 1994;14:5381-92.

7. Young LJ, Toloczko D, Insel TR. Localization of vasopressin (V1a) receptor binding and mRNA in the rhesus monkey brain. J Neuroendocrinol. 1999:11:291-7.

8. Lim MM, Bielsky IF, Young LJ. Neuropeptides and the social brain: potential rodent models of autism. Int J Dev Neurosci. 2005;23:235-43.

9. Lee RJ, Coccaro EF, Cremers H, McCarron R, Lu SF, Brownstein MJ, et al. A novel $\mathrm{V} 1 \mathrm{a}$ receptor antagonist blocks vasopressin-induced changes in the CNS response to emotional stimuli: an fMRI study. Front Syst Neurosci. 2013;7:100.

10. Poirier GL, Cordero Ml, Sandi C. Female vulnerability to the development of depression like behavior in a rat model of intimate partner violence is related to anxious temperament, coping responses, and amygdala vasopressin receptor 1a expression. Front Behav Neurosci. 2013;7:35

11. Guastella AJ, Kenyon AR, Unkelbach C, Alvares GA, Hickie IB. Arginine vasopressin selectively enhances recognition of sexual cues in male humans. Psychoneuroendocrinology. 2011:36:294-7.

12. Knafo A, Israel S, Darvasi A, Bachner-Melman R, Uzefovsky F, Cohen $L$, et al. Individual differences in allocation of funds in the dictator game associated with length of the arginine vasopressin 1a receptor RS3 promoter region and correlation between RS3 length and hippocampal mRNA. Genes Brain Behav. 2008;7:266-75

13. Tansey KE, Hill MJ, Cochrane LE, Gill M, Anney RJ, Gallagher L. Functionality of promoter microsatellites of arginine vasopressin receptor 1A (AVPR1A): implications for autism. Mol Autism. 2011;2:3.

14. Thibonnier M, Graves MK, Wagner MS, Chatelain N, Soubrier F, Corvol P, et al. Study of V(1)-vascular vasopressin receptor gene microsatellite polymorphisms in human essential hypertension. J Mol Cell Cardiol. 2000;32:557-64

15. Kim SJ, Young LJ, Gonen D, Veenstra-VanderWeele J, Courchesne R, Courchesne $E$, et al. Transmission disequilibrium testing of arginine vasopressin receptor 1A (AVPR1A) polymorphisms in autism. Mol Psychiatry. 2002;7:503-7.

16. Wassink TH, Piven J, Vieland VJ, Pietila J, Goedken RJ, Folstein SE, et al. Examination of AVPR1a as an autism susceptibility gene. Mol Psychiatry. 2004;9:968-72.

17. Yirmiya N, Rosenberg C, Levi S, Salomon S, Shulman C, Nemanov L, et al. Association between the arginine vasopressin 1a receptor (AVPR1a) gene and autism in a family-based study: mediation by socialization skills. Mol Psychiatry. 2006;11:488-94. 
18. Kantojärvi K, Oikkonen J, Kotala I, Kallela J, Vanhala R, Onkamo P, et al. Association and promoter analysis of AVPR1A in finnish autism families. Autism Res. 2015:8:634-9.

19. Procyshyn $\mathrm{TL}$, Hurd PL, Crespi BJ. Association testing of vasopressin receptor 1a microsatellite polymorphisms in non-clinical autism spectrum phenotypes. Autism Res. 2017;10:750-6.

20. Zhang R, Zhang HF, Han JS, Han SP. Genes related to oxytocin and arginine-vasopressin pathways: associations with autism spectrum disorders. Neurosci Bull. 2017:33:238-46.

21. Francis SM, Kim SJ, Kistner-Griffin E, Guter S, Cook EH, Jacob S. ASD and genetic associations with receptors for oxytocin and vasopressin-AVPR1A, AVPR1B, and OXTR. Front Neurosci. 2016;22;10:516. eCollection 2016.

22. Meyer-Lindenberg A, Kolachana B, Gold B, Olsh A, Nicodemus KK, Mattay V, et al. Genetic variants in AVPR1A linked to autism predict amygdala activation and personality traits in healthy humans. Mol Psychiatry. 2009;14:968-75.

23. Yang SY, Cho SC, Yoo HJ, Cho IH, Park M, et al. Family-based association study of microsatellites in the 5' flanking region of AVPR1A with autism spectrum disorder in the Korean population. Psychiatry Res. 2010;178:199-201.

24. Yang SY, Cho SC, Yoo HJ, Cho IH, Park M, Kim BN, et al. Association study between single nucleotide polymorphisms in promoter region of AVPR1A and Korean autism spectrum disorders. Neurosci Lett. 2010;479:197-200.

25. Lord C, Rutter M, Le Couteur A. Autism diagnostic interview-revised: a revised version of a diagnostic interview for caregivers of individuals with possible pervasive developmental disorders. J Autism Dev Disord. 1994;24:659-85.

26. Lord C, Rutter M, DiLavore PC, Risi S. Autism diagnostic observation schedule: manual. Los Angeles: Western Psychological Services; 2008.

27. Rutter M, Bailey AJ, Lord C. Social Communication Questionnaire (SCQ). Los Angeles: Western Psychological Services; 2003.

28. Kim JH, Shin MS. A study of reliability \& validity for the Korean version of Asperger Syndrome Diagnostic Scale. J Korean Acad Child Adolesc Psychiatry. 2005;16:98-105.

29. Constantino JN, Gruber CP. Social responsive scale (SRS): manual. LoS Angeles: Western Psychological Services; 2005.

30. Oh KJ, Lee H, Hong KE, Ha EH. K-CBCL. Seoul: Chung Ang Aptitude Publishing Co.; 1997.

31. Kim SK, Kim OK. Social maturity scale. 5th ed. Seoul: Chung Ang Aptitude Publishing Co.; 1995

32. Kim TL. Ewha-Vineland Adaptive Behavior Scales (EWHA-VABS). Seoul: Ewha Womans University; 1993.

33. Park K, Yoon J, Park H. The manual of Korean Educational Development Institute-Wechsler Intelligence Scale for Children-Revised (KEDI-WISC-R). Seoul: Korean Educational Development Institute; 1991.

34. Beaudet AL. Autism: highly heritable but not inherited. Nat Med. 2007;13:534-6.

35. Ronald A, Edelson LR, Asherson P, Saudino KJ. Exploring the relationship between autistic-like traits and ADHD behaviors in early childhood: findings from a community twin study of 2-year-olds. J Abnorm Child Psychol. 2010;38:185-96.

36. Wigger A, Sánchez MM, Mathys KC, Ebner K, Frank E, Liu D, et al. Alterations in central neuropeptide expression, release, and receptor binding in rats bred for high anxiety: critical role of vasopressin. Neuropsychopharmacology. 2004;29:1-14.

37. Wang SS, Kamphuis W, Huitinga I, Zhou JN, Swaab DF. Gene expression analysis in the human hypothalamus in depression by laser microdissection and real-time PCR: the presence of multiple receptor imbalances. Mol Psychiatry. 2008;13:786-99.

38. Nelson SB, Valakh V. Excitatory/inhibitory balance and circuit homeostasis in autism spectrum disorders. Neuron. 2015;87:684-98.

39. Freeman SM, Smith AL, Goodman MM, Bales KL. Selective localization of oxytocin receptors and vasopressin 1a receptors in the human brainstem. Soc Neurosci. 2017;12:113-23.

40. Young AM, Campbell E, Lynch S, Suckling J, Powis SJ. Aberrant NF-kappaB expression in autism spectrum condition: a mechanism for neuroinflammation. Front Psychiatry. 2011;2:27.

41. Schmitz ML, Baeuerle PA. Multi-step activation of NF-kappa B/Rel transcription factors. Immunobiology. 1995;193:116-27.

42. Tonhajzerova I, Ondrejka I, Mestanik M, Mikolka P, Hrtanek I, Mestanikova A, et al. Inflammatory activity in autism spectrum disorder. Adv Exp Med Biol. 2015;861:93-8.
43. Li X, Chauhan A, Sheikh AM, Patil S, Chauhan V, Li XM, et al. Elevated immune response in the brain of autistic patients. J Neuroimmunol. 2009; 207:111-6.

44. Naik US, Gangadharan C, Abbagani K, Nagalla B, Dasari N, Manna SK. A study of nuclear transcription factor-kappa B in childhood autism. PLoS One. 2011;6:e19488

45. Ziats MN, Rennert OM. Expression profiling of autism candidate genes during human brain development implicates central immune signaling pathways. PLoS One. 2011;6:e24691.

46. Skafidas E, Testa R, Zantomio D, Chana G, Everall IP, Pantelis C. Predicting the diagnosis of autism spectrum disorder using gene pathway analysis. Mol Psychiatry. 2014;19:504-10

47. Hu WW, Lai Y. Developing a predictive gene classifier for autism Spectrum disorders based upon differential gene expression profiles of phenotypic subgroups. N Am J Med Sci (Boston). 2013;6:1-9.

\section{Submit your next manuscript to BioMed Central and we will help you at every step:}

- We accept pre-submission inquiries

- Our selector tool helps you to find the most relevant journal

- We provide round the clock customer support

- Convenient online submission

- Thorough peer review

- Inclusion in PubMed and all major indexing services

- Maximum visibility for your research

Submit your manuscript at www.biomedcentral.com/submit
) Biomed Central 